\title{
Exploring Long Lifetime Routing (LLR) in ad hoc networks
}

\author{
Zhao Cheng \\ Department of Electrical and Computer \\ Engineering \\ University of Rochester \\ Rochester, NY 14627 \\ zhcheng@ece.rochester.edu
}

\author{
Wendi B. Heinzelman \\ Department of Electrical and Computer \\ Engineering \\ University of Rochester \\ Rochester, NY 14627 \\ wheinzel@ece.rochester.edu
}

\begin{abstract}
In mobile ad hoc networks, node mobility causes links between nodes to break frequently, thus terminating the lifetime of the routes containing those links. An alternative route has to be discovered once a link is detected as broken, incurring extra route discovery overhead and packet latency. A simple solution to reduce the frequency of this costly discovery procedure is to choose a long lifetime route carefully during the route discovery phase rather than a simple random shortest-path route scheme. This simple solution, although straightforward, requires investigation of several questions before it can be implemented. Specifically, how much effect does node mobility have on the link lifetime? Correspondingly, how much effect does it have on the route lifetime? How much lifetime extension can we achieve by using a long lifetime route compared to a randomly chosen shortest-path route? How much benefit can we obtain by using these longer lifetime routes, and what is the tradeoff of using them? By answering these questions, we gain enough insight to determine under what circumstances long lifetime routes are worth being discovered and implemented. In this paper, we first formulate the distribution of a link lifetime and correspondingly the distribution of a route lifetime. Then we present an algorithm to determine the longest lifetime routes at different route lengths. We experimentally compare these long lifetime routes with traditional random shortest-path routes and reveal the tradeoff of using long lifetime routes of different lengths. All the fundamental results will serve as the guideline for our future implementation of a distributed long lifetime routing protocol.
\end{abstract}

\section{Categories and Subject Descriptors}

C.2.2 [Network Protocols]: Routing protocols

\section{General Terms}

Design, Algorithms, Experimentation, Performance

Permission to make digital or hard copies of all or part of this work for personal or classroom use is granted without fee provided that copies are not made or distributed for profit or commercial advantage and that copies bear this notice and the full citation on the first page. To copy otherwise, to republish, to post on servers or to redistribute to lists, requires prior specific permission and/or a fee.

MSWiM'04, October 4-6, 2004, Venezia, Italy.

Copyright 2004 ACM 1-58113-953-5/04/0010 ...\$5.00.

\section{Keywords}

link lifetime distribution, Long Lifetime Routing (LLR)

\section{INTRODUCTION}

In an ad hoc network, there is no pre-existing fixed network architecture. Mobile nodes, typically with similar transmission and computational capabilities, cooperate by forwarding packets for nodes that are not in each other's direct transmission range. Routing protocols proposed for ad hoc networks can be roughly divided into two categories: tabledriven (proactive) and on-demand (reactive). Typical examples of table-driven protocols are DSDV [1] and OLSR [2]. These protocols require nodes to maintain a route table for all the other nodes so that a route is always available when a packet is ready to be transmitted. However, on-demand protocols attract more interest than table-driven protocols because they only initiate a route discovery process when a packet is ready to be transmitted. Without the necessity of persistent maintenance of a routing table, where shortest path algorithms are usually applied, on-demand protocols typically have lower routing overhead than table-driven protocols. Typical examples of these reactive protocols are DSR [3] and AODV [4].

Node mobility is one of the most important characteristics that affects the performance of MANETs. When a link breaks due to node mobility, the routes containing this link also become invalid. Therefore, an alternative route has to be discovered. This new discovery phase incurs networkwide flooding of routing requests and extended delay for packet delivery. To minimize the adverse impact of link breakage and corresponding route discoveries from node mobility, an intuitive method is to find routes with long lifetimes rather than choosing routes randomly. Traditional routing protocols usually result in finding shortest paths since the first route request to be responded to by the destination node tends to arrive following the shortest path. These shortest paths tend to contain links of long distances, which may break very soon. However, simply using more hops cannot alleviate the problem. This is because a route is composed of several links and the lifetime of a route actually equals the shortest lifetime among all the links in the route. Although using more hops may reduce the distance between links, the increasing number of hops also introduces more risk of route breakage. The effect of node mobility on link stability and route lifetimes has to be studied first to understand the limitations of using more links to form a 
route and the proper strategies for forming a long lifetime route.

Although finding long lifetime routes (LLRs) sounds promising in reducing routing overhead and unnecessary packet delay, the benefit is not free. Longer lifetime routes tend to contain more hops, and thus consume more energy to transport each packet. Also, the packet delay may increase compared to using a stable shortest path. Little research has been done in determining how much benefit we can obtain from those long lifetime routes and what are the costs. In our work, we design an algorithm to determine LLRs and compare the performance of these LLRs with that of random shortest-path routes. From the quantitative results, we conclude that there are several application scenarios where LLR can be applied to benefit the application.

The contributions of this paper are manyfold. First, we derive a closed form for link lifetime distribution and route lifetime distribution and statistically characterize the relationship between node mobility and link stability in section 3. Second, we propose an algorithm for determining the longest lifetime routes at different route lengths and therefore achieve the statistical results on the lifetime extension of LLRs in section 4. Third, we reveal the benefits of choosing long lifetime routes and the potential tradeoffs through extensive experiments in section 5. Also, we determine some suitable applications for LLRs based on the experimental results.

\section{OVERVIEW AND RELATED WORK}

Shortest-path routing is the most common algorithm in existing ad hoc routing protocols [3, 4]. However, as pointed out by [6], shortest path is not good enough for link stability, even for stable multi-hop wireless networks. In mobile ad hoc networks, links are even more fragile due to node mobility. A good metric to enable adaptive routing protocols, as pointed out by [7], is link duration, or in other words, link lifetime. Both theoretical and statistical studies have been done to discover the dependency of a link's residual lifetime with the link's age $[8,9]$. In our work, we reinvestigate the distribution of link lifetimes for some simplified mobility models and mainly focus on the factors that determine the distribution of a link lifetime. Although our analysis may not be able to represent all the lifetime distributions, which vary for different mobility models, it provides insight on the factors that affect a link's lifetime.

Several signal strength based routing protocols have been proposed such as ABR [10], SSA [11] and RABR [12]. The common idea behind these approaches is to prefer stable links or strongly connected links rather than transient links. The routing protocol, however, is concerned more with the route lifetime than individual link lifetimes. Therefore, choosing a long lifetime route rather than individual long lifetime links should be considered. Preemptive routing [13] attempts to determine an alternative route when it detects a possible link breakage from weak received signal strength. In contrast, in our work, we manage to discover a long lifetime route during the route discovery phase rather than to remedy the situation later. Despite this difference, our approach can be easily incorporated with preemptive routing to achieve even better performance.

Although link durations and link stability are studied extensively, there is little research on long lifetime route discovery. Discovering a strong link does not necessarily lead to discovering a long lifetime route since the other links extending from the end node of this strong link may be of very short link lifetime. Therefore, discovering a long lifetime route requires a higher level consideration than just from the link level. Choosing long lifetime routes that may be of longer lengths also raises an important question: how much benefit we can achieve from these long lifetime routes compared to random shortest-path routing and what is the tradeoff resulting from potentially increasing route lengths? To the best of our knowledge, this work is the first formal study to compare long lifetime routing schemes with random shortest-path routing schemes.

\section{LINK/ROUTE LIFETIME DISTRIBUTION}

In this section, we will first study the link lifetime distributions for some basic mobility patterns. We will provide the closed forms for one-dimensional scenarios and compare them with statistical results for two-dimensional scenarios. We draw several general conclusions about the relationship between node mobility and link stability. Afterwards, we will further discuss route lifetime distribution and explain how a route should be chosen to achieve the most lifetime extension in an ideal scenario.

\subsection{Link lifetime distribution}

The basic two questions to answer in this section are how node mobility affects the link lifetime distribution and what are the factors that affect link lifetime the most. One general assumption for the following analysis is that the transmission range of each node is one unit and the initial link distance $D$ between two nodes is uniformly distributed within $[0,1]$. This assumption matches the case where a route request packet may arrive at any time and the link distance may be of any value lower than the unit transmission range. Second, we simplify our analysis by assuming that the directions nodes move do not change before the link breaks. Although different mobility patterns may have nodes change directions, we can still assume that nodes do not change their directions of motion within a reasonable time period. These two assumptions simplify our analysis to enable us to answer the question posed earlier about the dominating factors of node mobility on the link lifetime distribution.

\subsubsection{The effect of number of mobile nodes}

Let us first look at the simplest one-dimensional space. Two nodes are separated by a distance of $D$ uniformly distributed in $[0,1]$. One node is moving with a speed $S$ uniformly distributed within $[0,1]$, either toward or away from the other node. After time $T$, their distance becomes larger than the unit transmission range and we say that the link is broken. What is the cumulative distribution function (cdf) $F_{T}(t)$ of the link lifetime $T$ ? Note that $F_{T}(t)$ can be also explained as the probability that a route is already broken at time $t$.

Due to space constraints, we only provide the closed form here and omit all the proofs. The cdf of the link lifetime for the above question is

$$
F_{T}(t)=\left\{\begin{aligned}
\frac{t}{4} & t<2 \\
1-\frac{1}{t} & t \geq 2
\end{aligned}\right.
$$

Notice that if the maximum speed is $S_{m}$ instead of 1 , we just need to replace $t$ with $S_{m} t$ since scaling the maximum speed $S_{m}$ up is actually equivalent to scaling the time down. 

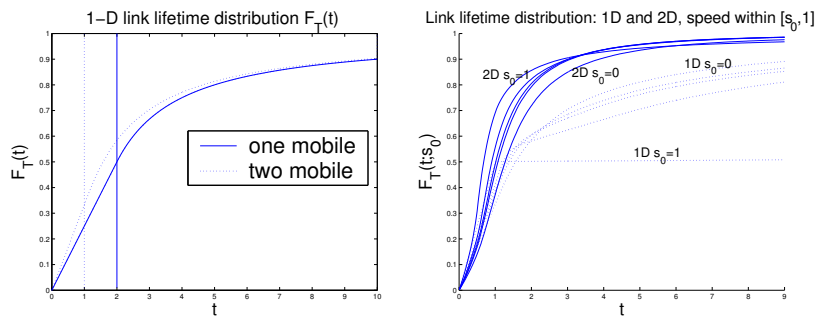

Figure 1: The effect of (a) the number of mobile nodes (b) the minimum speed on the cdf of link lifetime distribution. Neither of these two factors have much effect on $F_{T}(t)$.

If both nodes are moving instead of just one node, we can obtain the cdf of the link lifetime distribution as

$$
F_{T}(t)=\left\{\begin{aligned}
\frac{t}{3} & t<1 \\
1-\frac{1}{t}+\frac{1^{2}}{3 t^{2}} & t \geq 1
\end{aligned}\right.
$$

The cdf of $F_{T}(t)$ of the above cases for one mobile node and two mobile nodes are shown in the left plot of Fig. 1. A piecewise trend is shown: the link lifetime distribution shows a linear trend when time increases from zero; after a certain time, the curve increases very slowly. Also, the link lifetime distribution does not show significant difference between the case with one and two mobile nodes.

\subsubsection{The effect of the minimum speed}

Now we study the effect of the minimum speed by assuming that the speed of both nodes is uniformly distributed within $\left[s_{0}, 1\right]$ rather than $[0,1]$. The cdf of $F_{T}(t)$ in a onedimensional space becomes a complicated conditional piecewise function and non-trivial to show here. As for the twodimensional space, where both nodes may have a random speed direction within $[0,2 \pi]$, we cannot derive the final closed form. The involvement of the two more random angle variables makes the problem extremely hard to solve. However, we are able to obtain statistical results for the 2-D space through simulations.

In the right plot of Fig. 1, we compare the cdf of link lifetime for the 1-D and 2-D spaces for different values of the minimum node speed $s_{0}$. Two conclusions are drawn from this figure. First, node mobility in a 2-D space makes a link even easier to break than that in a 1-D space. In addition, unlike in the $1-\mathrm{D}$ case where nodes may be relatively static when they have the same speed, links in twodimensional space will eventually break after a certain time, even if nodes move with the same speed. Another interesting conclusion is that in two-dimensional space, the minimum speed $s_{0}$ does not have as much impact on the lifetime distribution as that in the one-dimensional case. In other words, only the maximum moving speed is the dominant factor of the link distribution. This trend simplifies our study since the link lifetime can be seen as only dependent on the maximum node speed.

\subsubsection{The effect of moving probability}

In the popular Random WayPoint model originally proposed in [3], nodes may pause for a certain time after reaching their destinations. To integrate this non-definite mobility into our model, we introduce a moving probability $p$.

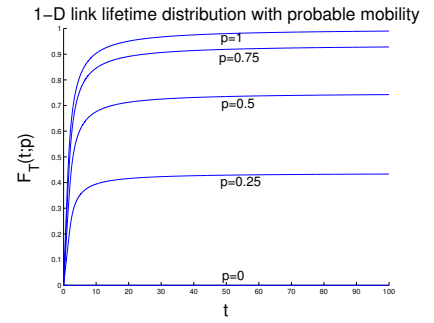

Figure 2: The cdf of link lifetime with different moving probability $p$.

Suppose nodes may move with a certain probability $p$ and may be static with probability $1-p$. The cdf of the link lifetime $F_{T}(t)$ becomes

$$
F_{T}(t ; p)=\left\{\begin{array}{rr}
-\frac{t}{6} p^{2}+\frac{p t}{2} & t<1 \\
\left(1-\frac{1}{t}+\frac{1}{3 t^{2}}-\frac{t}{2}\right) p^{2}+p \frac{t}{2} & 1<t<2 \\
\left(\frac{1}{t}-1+\frac{1}{3 t^{2}}\right) p^{2}+2 p\left(1-\frac{1}{t}\right) & 2<t<\infty \\
1 & t=\infty
\end{array}\right.
$$

The cdf of $F_{T}(t)$ with different moving probabilities $p$ is shown in Fig. 2. A similar piecewise linear trend is shown. However, unlike the insignificant difference between only one node moving and both nodes moving shown in Fig. 1, mobility probability $p$ does have considerable effect on the link lifetime distribution. It is obvious that the smaller the moving probability $p$, the longer lifetime a link tends to have. When both nodes are not moving $(p=0$,$) the link never$ breaks.

The two questions proposed at the start of this section can be answered now. First, the link lifetime distribution in all cases shows a two piecewise tendency. Since we are more interested in when a link is less likely to break, we can take the link breaking probability as linearly increasing with the link lifetime. Second, the only factor that has significant effect on the lifetime distribution is when both nodes may pause. Neither the minimum node speed nor only considering a single mobile node has much effect on link lifetime distribution. This conclusion matches the investigation from [14] that the number of network topology changes decreases rapidly when the pause time of the Random WayPoint (RWP) model increases. With these answers, we not only obtain an in-depth look of the effect of node mobility on link stability, we are also able to apply these results directly in the next section for the discussion of route lifetime distribution.

\subsection{Route lifetime distribution}

A route is composed of several links. The lifetime of the route is actually that of the link that has the shortest lifetime among all the links. Suppose we can choose the positions of the forwarding nodes that form the links in between the source and the destination node, what is the best strategy to form the route? More specifically, how many hops should we choose and where should these intermediate nodes be located so that we can obtain the optimal route lifetime distribution?

First of all, by intuition we want to choose all the intermediate nodes evenly spaced on the straight line between the source node and the destination node. We do not want any link to become the weakest link. Therefore, the remaining question is simple: how many intermediate nodes should we 

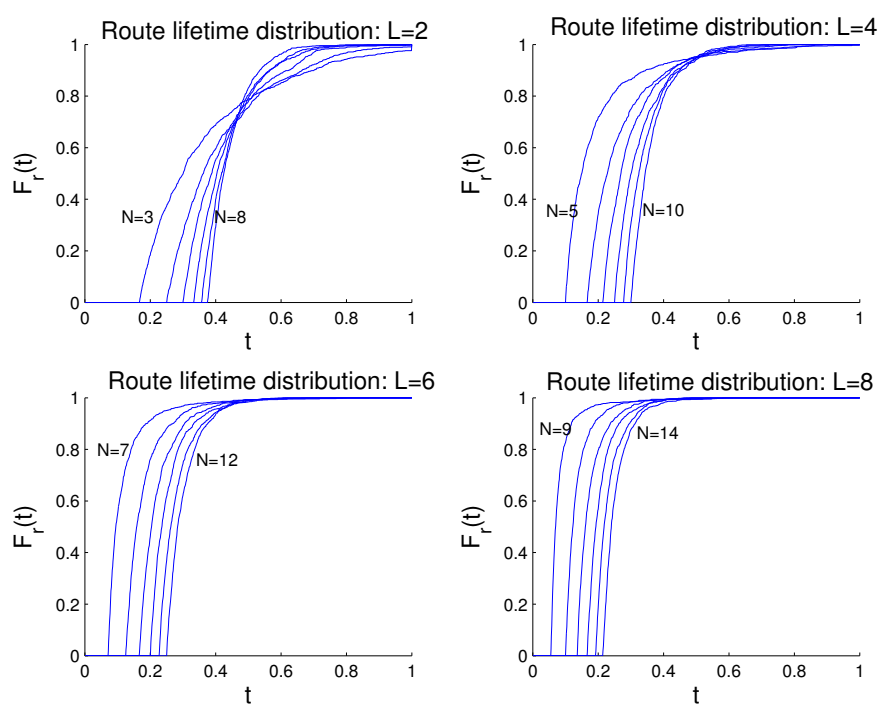

Figure 3: The cdf of route lifetime for different source-destination distance $L$ and number of links $N$.

insert in between the source and the destination? This is where the tradeoff comes in. If the fewest hops are used and the distance of links are chosen as large as too close to 1 , these links tend to break easily and the route lifetime will be reduced. However, if the distance of links are chosen too small, there will be too many links in the route. The route may also become unstable since once one link breaks, the entire route breaks. Also, from the routing perspective, long routes consume more energy for packet forwarding and reduce the network capacity.

Suppose the distance between the source node and the destination node is $L$. Suppose the source node wants to form a route composed of $N$ links where $N>L$, nodes are evenly spread in between the source and the destination and each link distance $D=\frac{L}{N}$. Since we already have the link lifetime distribution $F_{l}(t ; D)$ using the knowledge from the last section, we are able to express route lifetime distribution $F_{r}(t ; L, N)$ as

$$
F_{r}(t ; L, N)=1-\left(1-F_{l}\left(t ; \frac{L}{N}\right)\right)^{N}
$$

We experiment using a 2-D scenario where nodes have a constant speed of 1 with random speed directions. We tested the source-destination distance $L=\{2,4,6,8\}$ and the number of links $N=\{L+1, \cdots, L+8\}$. The route lifetime distributions are shown in Fig. 3 .

Each curve consists of two phases. Since the relative speed of two nodes is at most 2 , when $2 t<1-D$, it is impossible for links to break. Therefore $F_{r}(t)=0$ when $t<\frac{1-D}{2}$. After that period, $F_{r}(t)$ increases monotonically. The lifetime extension of using more hops, however, reduces when the number of hops increases. Let us take an example. When the route length is $L=4$, using 5 links will cause a route to break with probability $F_{r}(t)=0.6$ at time $t=0.16$ while using 6 links could extend the route lifetime to 0.24 for the same breaking probability. However, using 8 links can only extend the lifetime to 0.29 , which is a very limited improvement compared to using 6 links.
A long lifetime routing scheme can be implemented using two different methods. The first method is stochastically based using the knowledge from Fig. 3. Routes of longer length than the shortest path may be chosen since they have a smaller route breaking probability after the same time period. This stochastic method is simple in that it does not require individual link status, it is based on the route length. However, this stochastic scheme may not be effective in finding long lifetime routes in reality. Remember that the conclusions drawn earlier are based on an ideal scenario where intermediate nodes can be arbitrarily chosen in between the source and destination. This is only close to possible for a very dense network, which is not the case for most ad hoc networks. Therefore, an alternative deterministically based method should be considered. Nodes may choose a route based on the quality of each link. This deterministic scheme unavoidably requires link quality estimation, as was done in the signal strength based routing schemes mentioned in Section 2. Although this signal strength estimation adds to the protocol complexity, we believe that it is much more effective in determining a long lifetime route and the overhead will be compensated by the extension in route lifetime.

\section{LLR SELECTION ALGORITHM}

In the previous section, we investigate the distributions of link lifetime and route lifetime based on some fundamental mobility models. The study on the route lifetime distributions tells us that despite the higher complexity, a deterministic routing design for LLR is more suitable for real life scenarios than a probabilistic scheme. In this section, we will study how to determine long lifetime routes between a pair of nodes given a random network snapshot. We first provide a polynomial time algorithm to determine the longest lifetime routes at different route lengths from all the possible routes between the source and the destination. Using this algorithm, we are able to gather statistical results on the achievable maximum route lifetime improvement in random networks.

Here, we put $N$ nodes randomly in a circle of unit radius centered at location $(0,0)$. A source node $\mathbf{S}$ is placed at $\left(x_{s}, 0\right)$ and a destination node $\mathbf{D}$ is placed at $\left(x_{d}, 0\right)$. All the nodes have the same transmission range $R_{t}$. Nodes are assigned a speed uniformly distributed in $\left[s_{\min }, s_{\max }\right]$ and a moving direction uniformly distributed in $[0,2 \pi]$. At time $0, \mathbf{S}$ chooses a route to $\mathbf{D}$, and at time $T$, the route is broken. We are interested in the statistical results of following metrics.

1. The longest route lifetime and its route length.

2. The longest route lifetime of the shortest path. This is the best case among all the shortest paths.

3. The shortest route lifetime of the shortest routes. This is the worst case for all the shortest paths.

4. The longest route lifetime at route lengths between the longest and the shortest route length and their corresponding lifetimes. This metric will be further studied in the next section to compare with the lifetime of random shortest-path routes.

The following algorithm is proposed to discover qualified long lifetime routes within a polynomial time. The basic idea of this algorithm is to first sort all the links based on their weights: link lifetime in this case. Then we add the links in a descending order and adjust route lengths between each pair of nodes one by one. Meanwhile, we keep a record 


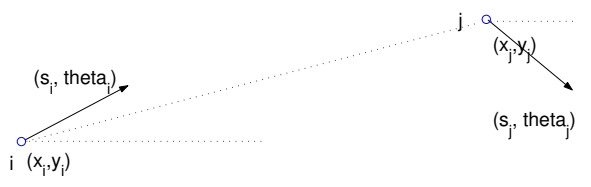

Figure 4: The geometry to calculate link lifetime.

for all the route length changes and their corresponding lifetime changes for the source-destination pair. After every link is added, we will have a complete record of any lifetime changes between the source-destination pair.

We are only interested in the lifetime and length of the path between the source node $\mathbf{S}$ and the sink node $\mathbf{D}$. The arc set $A$ is sorted in descending order by the lifetime $c[i, j]$ of the link composed of nodes $i$ and $j$. Given a snapshot of the network, if the link distance between node $i$ and node $j$ is shorter than the transmission range, their link lifetime $c[i, j]$ is determined as in Fig. 4 and equation 5.

$$
\begin{aligned}
D^{2}(t)= & {\left[\left(x_{i}+s_{i} \cos \theta_{i} t\right)-\left(x_{j}+s_{j} \sin \cos \theta_{j} t\right)\right]^{2} } \\
& +\left[\left(y_{i}+s_{i} \sin \theta_{i} t\right)-\left(y_{j}+s_{j} \sin \theta_{j} t\right)\right]^{2}
\end{aligned}
$$

By solving $D(t)=R_{t}$, we will have the link lifetime $t$ assigned to $c[i, j]$. Notice that when the network snapshot is given, all the node location and speed information is deterministic.

We denote an edge as $e$ or a link between node $i$ and $j$ as $e[i, j]$ if node $i$ and $j$ are connected. $d[i, j]$ is the hop distance between nodes $i$ and $j . d_{\text {prev }}$ is the last route length recorded between the pair. The Long Lifetime Route (LLR) selection algorithm is shown in Algorithm 1.

Data: $A$, initial $c[i, j]$ for each link

Result: Record of the longest lifetime achievable for routes with different hop distances $\{d[\mathbf{S}, \mathbf{D}], c[\mathbf{S}, \mathbf{D}]\}$

begin

$$
\begin{aligned}
& S:=\emptyset ; \bar{S}:=A ; d_{\text {prev }}=\infty ; \\
& \text { for all node pairs }[i, j] \in N \times N \text { do } \\
& \mid d[i, j]:=\infty ; \operatorname{pred}[i, j]:=0 ; \\
& \text { end } \\
& \text { for all nodes } i \in N \text { do } d[i, i]:=0 \text {; } \\
& \text { while }|S| \neq A \text { do } \\
& \text { let } e[i, j] \in \bar{S} \text { for which } c[i, j]=\max \{c(e), e \in \bar{S}\} \text {; } \\
& S:=S \bigcup\{[i, j]\} ; \bar{S}:=\bar{S}-\{[i, j]\} ; \\
& d[i, j]=d[j, i]=1 \\
& \text { for each }[m, n] \in N \times N \text { do } \\
& \text { if } d[m, n]>d[m, i]+d[i, j]+d[j, n] \text { then } \\
& d[m, n]:=d[m, i]+d[i, j]+d[j, n] \text { and } \\
& \operatorname{pred}[m, n]:=i \text {; } \\
& \text { end } \\
& \text { if } d[m, n]>d[m, j]+d[j, i]+d[i, n] \text { then } \\
& d[m, n]:=d[m, j]+d[j, i]+d[j, n] \text { and } \\
& \operatorname{pred}[m, n]:=j \text {; } \\
& \text { end } \\
& \text { end } \\
& \text { if } d[\boldsymbol{S}, \boldsymbol{D}]<d_{\text {prev }} \text { then } \\
& d_{\text {prev }}=d[\mathbf{S}, \mathbf{D}] \text { and record }\{d[\mathbf{S}, \mathbf{D}], c[\mathbf{S}, \mathbf{D}]\} \\
& \text { end } \\
& \text { end }
\end{aligned}
$$$$
\text { end }
$$

Algorithm 1: LLR selection algorithm.
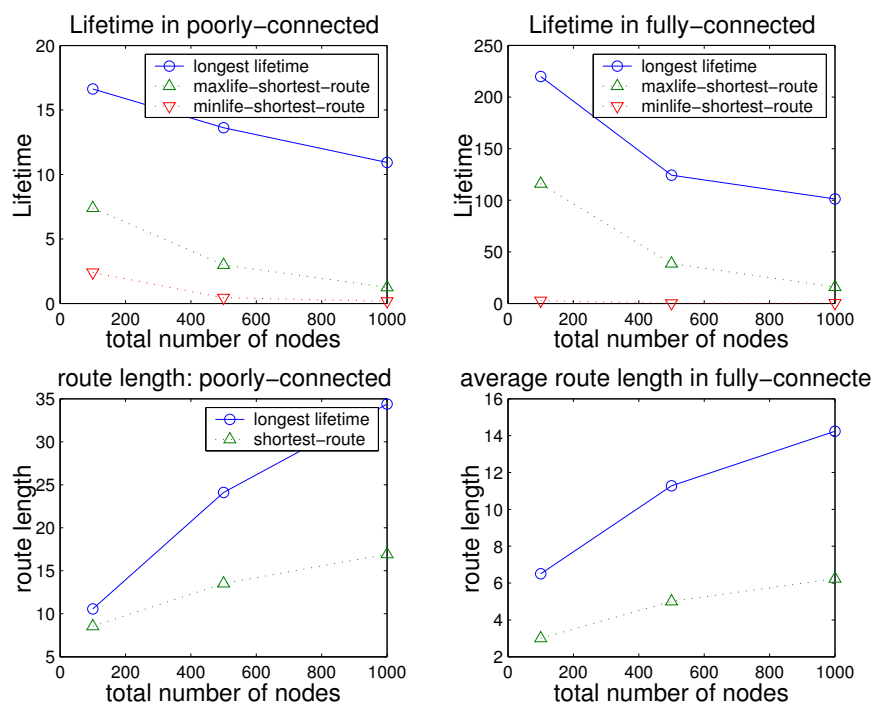

Figure 5: The impact of network size. The number of nodes is varied from 100 to 1000. Poorly connected $(\mathrm{C}=1)$ and fully connected networks $(\mathrm{C}=\mathbf{5 . 1 7 7 4})$ are shown.

The overall complexity of this algorithm is $O\left(|A| N^{2}\right)$. After running the algorithm, we have a record list composed of elements $\{d[\mathbf{S}, \mathbf{D}], c[\mathbf{S}, \mathbf{D}]\}$, which is the longest lifetime route at different hop distances between the source and the destination node. Although this algorithm may not be the most efficient, it is good enough to find all the statistical results we need. Note that our LLR algorithm requires global knowledge and hence is not suitable for implementation. We use this algorithm only to investigate the performance of LLRs.

\subsection{Statistical results}

We ran the LLR selection algorithm using 50 different scenarios in a unit circle and averaging the results. We study the impact of the following network parameters on the route lifetime and route lengths: network size by varying $N$ for the number of nodes, network connectivity by varying the transmission range $R_{t}$, node speed by varying the minimum speed of the nodes $S_{\min }$ and the source-destination distance by varying the source location $\left(x_{s}, 0\right)$ and the destination location $\left(x_{d}, 0\right)$. We adjust the network connectivity by calculating $R_{t}=\sqrt{\frac{C \log (N)+1}{N}}$ using different connectivity in$\operatorname{dex} C$ [15]. We vary $C$ from $\{1,3,5.1774\}$, which represents poor connectivity, moderate connectivity and full connectivity, respectively.

First we study the impact of the network size by varying the total number of nodes $N$ from $\{100,500,1000\}$. Both poorly connected and fully connected networks are studied. The connectivity is affected by the transmission range $R_{t}$. Other parameters are $S_{\min }=0, x_{s}=-0.5$ and $x_{d}=0.5$.

Results are shown in Fig. 5. In both poorly and fully connected networks, a good route can last several times longer than the best case of the shortest-path route. The shortest path may last for a very short time if the worst route is chosen.

We also tested the impact of other network parameters 


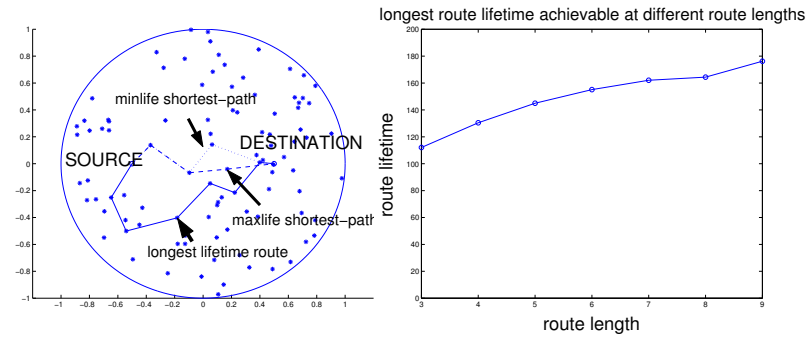

Figure 6: A snapshot of how the longest lifetime route, the best shortest-path route and the worst shortest-path route are formed is shown on the left. The longest lifetime achievable for routes of different route lengths are shown on the right. A linear tendency is shown.

such as the minimum speed, connectivity and the initial distance between the source and destination. Increasing the minimum speed from 0 to $\frac{S_{\max }}{2}$ may reduce the longest lifetime greatly. However, increasing from $\frac{S_{\max }}{2}$ to $S_{\max }$ does not affect the lifetime that much. This is because for a larger $S_{\text {min }}$, the effect of speed is partly counteracted by the random moving directions. Increasing connectivity will linearly increase the lifetime since more choices are available. It will also reduce the route length for the same reason. Increasing the initial distance between the source and destination does not affect the lifetime too much, only the route length increases linearly. In all of the above cases, LLR has a much longer route lifetime than that of the shortest-path routes.

We show on the left plot of Fig. 6 an example of how the longest lifetime route, the best shortest-path route and the worst shortest-path route are formed. In general, we find that the route lifetime can be significantly extended in most scenarios if we allow a much longer route length. However, even the route with the best shortest path length demonstrates a much better lifetime performance than that of the worst shortest path.

In the above results, we only show the longest lifetime route and the route that achieves the maximum lifetime among all the shortest-path routes. However, although the longest lifetime route is able to avoid some short lifetime links, it usually ends up with a long route length and an unexpected route itinerary (see Fig. 6). Thus, in order to make the study practical for our later implementation, we are willing to find some routes with a longer lifetime while not requiring too many extra hops compared to the shortest path.

Using our LLR selection algorithm, we recorded the longest lifetime achievable at different route lengths and show it in the right plot of Fig. 6. As can be seen, the longer the route length, the longer the lifetime achievable, and the relation shows a linear tend. The tradeoff of using these long hopdistanced routes and the benefit from their long lifetime will be fully explored next.

\section{SIMULATION RESULTS}

Although we have determined how we may extend the route lifetime linearly by using longer route lengths, we have still not discussed the benefit of the extended lifetime and the tradeoff of using longer routes. Intuitively, Long
Lifetime Routes (LLR) are more stable than Shortest-Path Routes (SPR) in terms of packet delivery ratio and packet latency. Using a long lifetime route, packets can be delivered within certain delay constraints and without any packet loss as long as there is no congestion along the path. In contrast, relatively frequent link breakage from SPRs may cause packet drops and may jitter the packet delay. Using LLRs, less frequent route maintenance is required, which is usually a costly flooding-based procedure. However, the drawbacks of LLRs are also obvious due to their longer route length. First of all, more energy will be consumed for delivering the same packet if there are more hops in the route. Although LLRs require fewer flooding-based procedures for route discovery, the energy efficiency is highly dependent on the utilization of the path. When more traffic is to be carried within a given time, the maintenance overhead from SPRs will become smaller compared to that of packet forwarding, and LLR may not exhibit better efficiency since packets have to be forwarded more hops than with SPR. Another adverse effect of using longer routes with LLR is that it may bring more potential collisions and congestion to the network. The overall capacity of the network may also decrease from the longer route lengths.

In this section, we will experimentally investigate the performance of LLR with different number of hops compared to that of SPR. DSR is used as the carrier routing protocol for the comparisons. The goal of this study is to quantitatively determine under what circumstances should LLR be applied and what criteria should guide our future LLR design.

\subsection{LLR intra-comparisons}

Several traditional routing protocol metrics are of interest to us: packet delivery ratio, packet delivery latency, energy consumption and throughput. First, we will compare the performance of LLR at different traffic loads between a pair of nodes. We will find out how these metrics vary when we choose LLRs with different route lifetimes and different route lengths. We look at a fully connected network by randomly placing 100 nodes inside a network with unit radius centered at $(0,0)$. The transmission range of each node is 0.5 . We place the source node at $(-0.5,0)$ and the destination node at $(0.5,0)$. Nodes move with a speed uniformly distributed from $\left[0, S_{m}\right]$ in a random direction without stopping. As we mentioned earlier, the choice of $S_{m}$ only scales the lifetime of LLRs and does not affect their relative performance. 802.11 is used as the MAC layer protocol and the wireless bandwidth is $2 \mathrm{Mbps}$. The Ns-2 simulator is used.

We feed the source node packets of 64 bytes with a traffic rate from 1 packet per second to 300 packets per second. LLRs at different route lengths are found using the LLR selection algorithm from section 4 and are preset into the routing table of the source node. For each experiment, 50 scenarios are tested and the results are averaged.

Fig. 7 shows the performance of LLRs at different lengths. First of all, LLRs with longer route lengths show a decreasing packet delivery ratio compared with LLRs with shorter route lengths, especially when the feeding traffic rate exceeds a certain threshold. This threshold, as shown in the bottom right plot, is actually the capacity of this multi-hop route. In ad-hoc networks, neighboring nodes share the same wireless resources and may interfere with each other's transmissions. The longer the route length is, the more interference there is and the smaller the capacity of this route. For example, 
an LLR composed of 5 hops can only let 125 packets pass in one second. More packets fed into the route have to be dropped, and the packet delivery ratio for this higher traffic rate will decrease.

Both the latency and the energy cost show a bi-modal trend with the number of hops. If the traffic feeding rate is below the capacity, both the latency and the energy cost show a linear trend. However, once the traffic rate exceeds the capacity, collisions occur more frequently and latency increases dramatically due to the repetitive MAC layer backoffs from these collisions. The energy, instead, will not increase since the channel is already fully utilized.
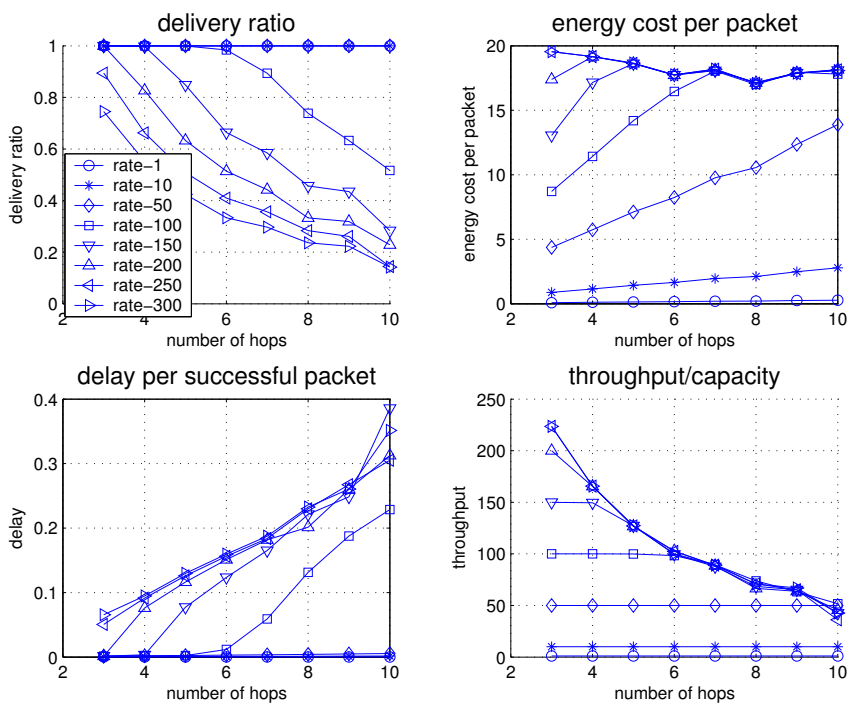

Figure 7: A comparison of LLRs with different route lengths and route lifetimes.

In summary, longer lifetime LLRs have longer route lengths, and therefore have less capacity and cost more energy to deliver a packet as long as the traffic to be carried does not exceed the capacity. However, considering that SPRs may require several route maintenance procedures during the lifetime of one LLR route, we need a reinvestigation of LLR to compare its performance with that of SPR.

\subsection{LLR vs. SPR using UDP}

We compare the performance of LLR and SPR using DSR as the carrier routing protocol and UDP as the transport protocol. We run the LLR until its lifetime ends and record its performance. Meanwhile, we run SPR for the same period and record its performance. Since we have different route lifetimes for different route length LLRs, we repeat the above procedure for all the possible route lengths of LLR. In this way, we are able to compare the performance of SPR with that of LLR at different route lengths.

The simulation results from Fig. 8 show that for both high and low traffic rates, LLR exhibits a stable packet delivery ratio and packet delay since the routes are less likely to become invalid. Also, LLR shows a higher energy efficiency for low traffic rates because SPR wastes much energy in finding new routes. However, when the traffic rate is high, SPR may be more energy efficient since the extra cost from route maintenance is very small compared to the energy consumed
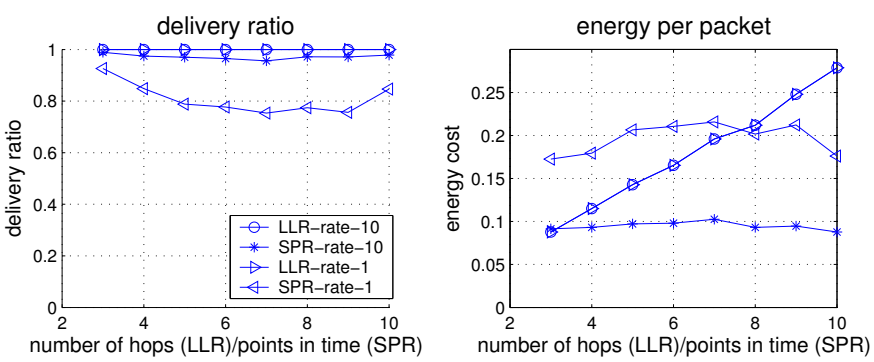

delay per successful packet
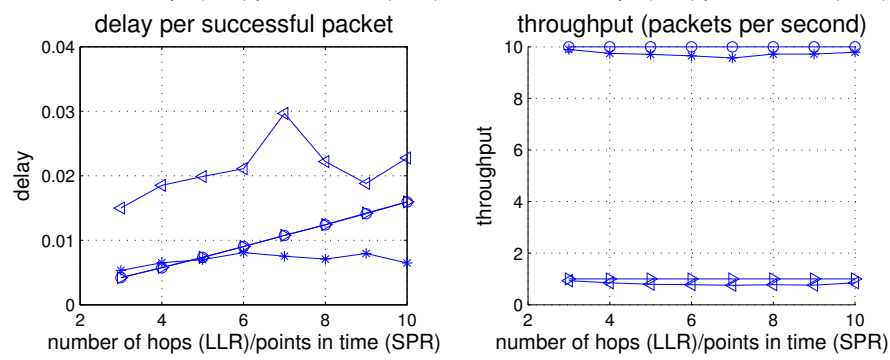

Figure 8: The performance of LLR with different route lifetimes at different hop distances compared with the SPR performance in the corresponding period. UDP is used as the transport protocol, and the traffic rate is 1 or 10 packets per second.

in packet forwarding, while LLR may forward a packet using a route with a longer hop distance.

\subsection{LLR vs. SPR using TCP}

We use TCP as the transport layer and redo the above simulations. The simulation results in Fig. 9 show that TCP performs poorly for SPR, especially after the route breaks. Several reasons cause this behavior, from TCP and DSR respectively. First, when a TCP acknowledge packet is not received from the destination, TCP will assume the channel is congested and will perform a slow start. Second, the multiple route caching scheme in DSR leads to long discovery latency since when the original path fails, a node has to try all the remaining route caches, which are very likely to be invalid already. The unsynchronization of the backoff scheme in DSR and the retransmission timeout scheme in TCP further increases the delay of route discovery. Therefore, it is very crucial for us to provide a better route discovery scheme to discover a long lifetime route so that we are able to improve the overall performance without modifying the existing ad hoc network architecture.

\subsection{Where to use LLR}

Based on the experimental results of LLR, the applications and environments for which LLRs are beneficial can be determined. The first application to use LLR is to find an LLR with the shortest route length, or we may find onehop longer LLR with an even longer route lifetime. In this way, we are able to provide stable routes without deteriorating energy efficiency and packet latency much.

LLR is also suitable for applications that require a long session but have very low traffic. Telnet is an example of this type of application. In this case, LLRs with moderate route lengths can be used while still being able to be more energy efficient and deliver more packets than SPRs. 


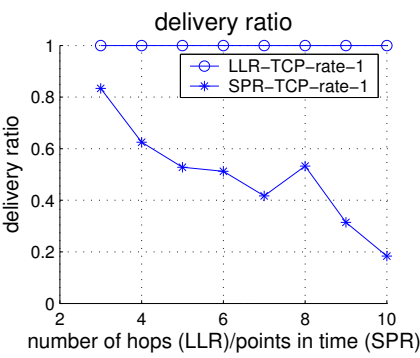

delay per successful packet
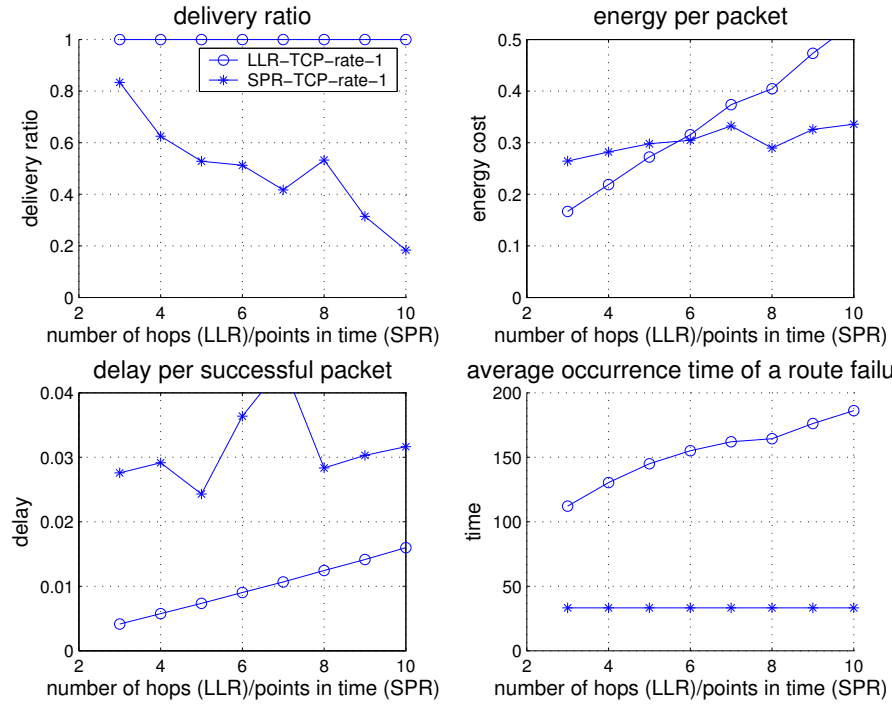

average occurrence time of a route failure

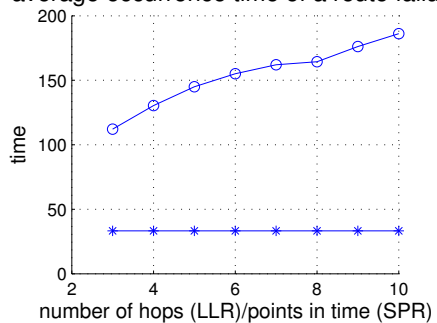

Figure 9: The performance of LLR with different route lifetimes at different hop distances compared with the SPR performance in the corresponding period. TCP is used as the transport layer. The traffic rate is 1 packet per second.

For a heavier traffic, LLR does not show any advantage in terms of energy efficiency since it tends to have more hops than SPRs. However, it provides a stable route that is very crucial for QoS-based applications. Therefore, LLRs may serve as backup for common SPRs. For example, for a realtime voice application, while discovering an SPR, an LLR can be discovered as well. When the SPR is expected to become invalid, the LLR can be utilized while performing a new SPR discovery. In this way, the real-time application will not be interrupted and certain QoS requirements can be satisfied. Simply finding several SPRs and using one as the primary route and the rest as backups, as is done in DSR, is not good for QoS-based applications since when the primary route becomes invalid, it is probable that the backup routes are already invalid or they are going to break soon.

\section{CONCLUSIONS AND FUTURE WORK}

In this paper, we first study the distributions of the link lifetime and route lifetime in different scenarios. The analysis indicates that simply increasing the route length by choosing short initial link distances may not be effective in extending the route lifetime. Although the design of distributed LLR protocols is not implemented, our work provides information on what applications can benefit from LLR and shows that link lifetime estimation is unavoidable for the design of an LLR protocol.

Our further research on designing LLR protocols is mostly composed of two parts. The first part is signal strength history based link lifetime estimation. Unlike most of the signal strength based estimation schemes that use ideal wireless propagation models, a more realistic wireless propagation model with both fast and slow fading will be taken into account. To reduce the adverse effects from fast fading, signal histories are required. The second part is to design a distributed LLR protocol. A possible distributed solution is to associate the RREQ packet forwarding time with the link lifetime. This mechanism will be fully evaluated.

\section{REFERENCES}

[1] C. Perkins and P. Bhagwat. "Highly Dynamic Destination-Sequenced Distance-Vector Routing (DSDV) for Mobile Computers," In Proc. of the ACM SIGCOMM, October 1994.

[2] P. Jacquet, P. Multethaler, A. Qayyum, A. Laouiti, L. Viennot, and T. Clausen, "Optimized link state routing protocol," Internet Draft, http://www.ietf.org /internet-drafts/draft-ietf-manet-olsr-04.txt

[3] D.B.Johnson and D.A.Maltz. Mobile Computing, Chapter Dynamic source routing in ad hoc wireless networks, pages 153-181. Kluwer Academic Publishers, Imielinski and Korth edition, 1996.

[4] C.Perkins and E.M.Royer. "Ad hoc on-demand distance vector routing" Proceedings of IEEE WMCSA'99, pp. 90-100, Feb. 1999.

[5] V. D. Park and M. S. Corson, "A highly adaptive distributed routing algorithm for mobile wireless networks," In Proc. of IEEE INFOCOM , 1997.

[6] D. De Couto, D. Aguayo, B. Chambers, and R. Morris, "Performance of multihop wireless networks: Shortest path is not enough," In Proc of HotNetsI, New Jersey, USA, Oct. 2002.

[7] J. Boleng, W. Navidi, and T. Camp, "Metrics to Enable Adaptive Protocols for Mobile Ad Hoc Networks," Proc of ICWN 02, pp. 293-298, 2002

[8] A. Bruce McDonald and Taieb Znati, "A Path Availability Model for Wireless Ad-Hoc Networks," in Proceedings of IEEE WCNC '99., New Orleans, LA.

[9] M Gerharz, C.D.Waal, M Frank and P Martini, "Link Stability in Mobile Wireless Ad Hoc Networks," Proc. of the 27th IEEE LCN, 2002.

[10] C.K. Toh, "Associativity-Based Routing for Ad HocMobile Networks," International Journal on WirelessPersonal Communications, Vol.4, No. 2, 1997.

[11] Rohit Dube, Cynthia D. Rais, Kuang-Yeh Wang, andSatish K. Tripathi, "Signal stability-based adaptive routing(SSA) for ad hoc mobile networks," IEEE Personal Communications, 4(1):36-45, February 1997

[12] S. Agarwal, A. Ahuja, J.P. Singh, and R. Shorey, "Route-Lifetime Assessment Based Routing (RABR) Protocol for Mobile Ad-Hoc Networks," Proc. of ICC, June 2000, pp. 1697-1701.

[13] T. Goff, N. B. Abu-Ghazaleh, D. S. Phatak and R. Kahvecioglu, "Preemptive Maintenance Routing in Ad Hoc Networks", MOBICOM 2001, pp. 43-52.

[14] T. Camp, J. Boleng and V. Davies, "A Survey of Mobility Models for Ad Hoc Network Research," WCMC: Special issue on Mobile Ad Hoc Networking: Research, Trends and Applications, vol 2, pp. 483-502.

[15] Feng Xue and P. R. Kumar, "The number of neighbors needed for connectivity of wireless networks" Manuscript, 2002. Available from http://black1.csl.uiuc.edu/prkumar/postscript files.html

[16] G Holland and N. H. Vaidya, "Analysis of TCP Performance over Mobile Ad Hoc Networks," Mobile Computing and Networking, pp. 219-230, 1999. 